\title{
A case of eosinophilic meningitis following monitor lizard meat consumption, exacerbated by anthelminthics
}

\author{
MDP Hidelaratchi ${ }^{1}$, MTM Riffsy $^{2}$ and JC Wijesekera ${ }^{3}$
}

(Index words: Angiostrongylus cantonensis, anthelminthics, monitor lizard)

\section{Introduction}

We report a case of eosinophilic meningitis following the consumption of monitor lizard meat (Varnus bengalensis). The history, clinical features and the cerebrospinal fluid (CSF) findings were suggestive of an infection with Angiostrongylus cantonensis, a nematode parasite of the rat lung. The exacerbation which occurred following the treatment with anthelminthics is emphasised.

\section{Case report}

A previously healthy 16-year old boy was admitted to a medical unit at the National Hospital of Sri Lanka with vague ill health of 10 days, intermittent low grade fever, severe headache, and paraesthesiae of legs. He did not give a history of neck pain, fits or symptoms suggestive of a focal lesion.

Ten days before the admission, he had consumed monitor lizard meat and although initially it was followed by abdominal discomfort and vomiting he had eaten the remainder by evening. He denied the consumption of seafood such as crabs, prawns or fish and green leafy vegetables.

He was afebrile, and apart from mild neck stiffness, the systemic examination was unremarkable. On admission, following the clinical diagnosis of bacterial meningitis, he was started on intravenous cephalosporin, but failed to show improvement.

Investigations revealed a peripheral blood leucocytosis with marked eosinophilia (white cell count $-16.2 \times 10^{9} / \mathrm{L}$, neutrophils $52 \%$, lymphocytes $20 \%$, eosinophils $27 \%$, monocytes 1\%). Platelets, haemoglobin, urine analysis, erythrocyte sedimentation rate, serum electrolytes, and blood urea were normal. Filarial antibody test, blood for malarial parasite, Mantoux test, stools for ova and cysts were negative. The CSF analysis showed clear, colourless fluid, containing high protein $(120 \mathrm{mg} / \mathrm{dL})$ with an increased cell count $\left(384 / \mathrm{mm}^{3}\right)$ and the smear showed abundant eosinophils with few lymphocytes. Organisms and acidfast bacilli were not detected. At this stage, a diagnosis of eosinophilic meningitis was made. The antibiotic was omitted and anthelminthic, albendazole was commenced.

Within 24 hours of starting the anthelminthic, a marked deterioration in sensorium was apparent and he was transferred to the Neurology Unit. The electroen- cephalogram showed frontal intermittent delta waves suggestive of raised intracranial pressure and the contrast computed tomography (CT) of brain was unremarkable. A repeat CSF analysis showed total cell count of $560 / \mathrm{mm}^{3}$ with over $50 \%$ eosinophils. This was confirmed with Leishman stain. Further haematological and biochemical investigations, including blood culture, antibody against Toxoplasma gondii, c-ANCA, p-ANCA, Venereal Disease Research Laboratory, and HIV antibody assay were negative.

Following the exacerbation, anthelminthics were omitted. The patient was started on intravenous dexamethasone $8 \mathrm{mg}$, eight hourly and he underwent two therapeutic lumbar punctures. The deterioration continued for 2 days but gradually a slow, steady improvement was observed within a week. With recovery, oral dexamethasone was started and subsequently tapered over 4 weeks.

The patient was reviewed following discharge, initially fortnightly and thereafter at monthly intervals for 8 months. The repeat CSF analysis and the serial complete blood counts were normal and the patient remained asymptomatic.

\section{Discussion}

The commonest infective cause of eosinophilic meningitis worldwide is by the nematode parasite, Angiostrongylus cantonensis. Humans are infected by accidental ingestion of the third stage larvae in raw or inadequately cooked intermediate hosts, such as snails, slugs or paratenic (carrier) hosts, including fish, amphibians, reptiles, crustaceans, or by eating vegetables and green leaves contaminated with larvae. The larvae of A. catonensis are inherently neurotropic and following ingestion by humans, they migrate to both central nervous system (CNS) and eye.

In the literature search we came across only a few case reports of eosinophilic meningitis following consumption of raw or partially cooked monitor lizard (Varnus bengalensis) meat [1,2]. This is the first case report of a similar incidence in Sri Lanka, although eosinophilic meningitis has been reported earlier [3]. Consumption of monitor lizard meat is a well known delicacy in our island. These lizards feed on snails, beetles, crabs, frogs, rodents and vegetables.

\footnotetext{
${ }^{1}$ Registrar in Medicine, ${ }^{2}$ Resident Neurologist, ${ }^{3}$ Neurologist, Institute of Neurology, National Hospital of Sri Lanka. Regent Street, Colombo 10, Sri Lanka.

Correspondence: MDPH, e-mail: <priyangika@sltnet.lk>. (Competing interests: none declared).
} 
The predominant symptom in our patient was an acute severe headache with paraesthesiae of extremities. This is the commonest symptom of cerebral angiostrongyliasis. Other symptoms with which these patients may present are visual loss, blepharospasms and transient cranial nerve palsies, especially of the seventh nerve. Fever, however, is an uncommon complaint.

Our patient was diagnosed on the history of ingestion of partially cooked monitor lizard meat, clinical features and the presence of CSF pleocytosis with over $50 \%$ of eosinophils. Paraesthesiae or hyperaesthesiae supports this diagnosis as these symptoms are unusual in other forms of meningitis.

Diagnosis is further aided by enzyme-linked immunosorbent assay (ELISA) to detect the antibodies to A. cantonensis and a more specific serological test to detect antibodies against the $31-\mathrm{kD}$ antigen of $A$. cantonensis [4]. Chye and others have recently described an even more promising technique for the detection of $A$. cantonensis antigens in serum samples, using Immuno-PCR [5]. These serological tests are not available in Sri Lanka.

The CSF was analysed for the presence of parasites on all occasions the patient underwent lumbar puncture, but could not be detected. The demonstration of parasites is confirmatory.

The changes in the CT scan of brain in cerebral angiostrongyliasis can vary from being normal to nonspecific findings. The CT imaging done on two occasions in our patient was normal. The magnetic resonance imaging (MRI) findings in CNS infection with A. cantonensis have been reported to be non-specific, ranging from normal to leptomeningeal enhancement, ventriculomegaly, punctuate area of abnormal enhancement, and hyperintense signal lesions on T2-weighted images [6].

Following the diagnosis of eosinophilic meningitis in our patient, albendazole was started, but this led to an exacerbation of his condition. However, albendazole and levamisole had been used in Taiwan and China with apparently good results [7,8]. A similar exacerbation, as seen in our patient, was reported from New Hebrides (Republic of Vanuatu), where two patients were treated with thiabendazole [9]. In a study on the outbreak of eosinophilic meningitis caused by Angyiostrongylus cantonensis in travelers returning from the Caribbean, anthelminthics had been withheld considering the theoretical possibility of exacerbation following the death of larvae in the central nervous system [10]. Many experimental studies on drug treatment of Angiostrongylus species have been carried out in mice and rats [11]. The place of anthelmenthics in A. cantonensis infection in humans will remain a controversy until a randomised controlled study is conducted.

The optimal symptomatic management of eosinophilic meningitis secondary to A. cantonensis infection is also not known. Serial therapeutic lumbar punctures and corticosteroids have been reported to improve the symptoms
[10]. A 2-week course of prednisolone $60 \mathrm{mg} /$ day has been recommended by Chotmongkol and others following their study on corticosteroid treatment for eosinophilic meningitis [12].

In our case report we wish to emphasise the need of being cautious when using anthelminthics in cerebral angiostrongyliasis, as there are no trial data on humans to support the efficacy. Although case reports on monitor lizards being paratenic hosts for A. cantonensis infection are few in number, awareness of this possibility will be of benefit for those who indulge in such delicacies.

\section{Acknowledgement}

We acknowledge Dr. Senaka Bandusena (Senior Registrar, Neurology) and Dr. Sagarika Samarasinghe (Parasitologist, MRI ) for literature search and advice.

\section{References}

1. Jitpimolmard S, Chotmongkol V, Morakote N. Eosinophilic meningitis caused by eating monitor lizard (Varanus bengalensis): a report of 6 cases from 2 outbreaks (abstract). In: Proceedings of the 8th Annual Meeting of the Royal College of Physicians of Thailand, 1992. Royal College of Physicians of Thailand, Bangkok; 1992: 150.

2. Kanpittaya J, Jitpimolmard S, Tiamkao S, Mairiang E. MR findings of eosinophilic meningoencephalitis attributed to Angiostrongylus cantonensis. American Journal of Neuroradiology 2000; 21: 1090-4.

3. Alibhoy AT, Senanayake B, Fernando MAH, Amerasekera HSU, Wijesekera JC. A case of eosinophilic meningitis. Ceylon Medical Journal 1999; 44: 173-4.

4. Eamsobhana P, Mak JW, Yong HS. Development of specific immunodiagnosis for human parastrongyliasis. In: Eamsobhana P, ed. Molecular Biology and Immunology in the Diagnosis of Parasitic Diseases with Emphasis on Malaria. Bangkok: Southeast Asian Medical Information Center 1996: 159-65.

5. Chye SM, Lin SR, Chen YL, Chung LY, Yen CM. ImmunoPCR for detection of antigen to Angiostrongylus cantonensis circulating fifth-stage worms. Clinical Chemistry 2004; 50: 51-7.

6. Tsai HC, Liu YC, Kunin CM, Lai PH, Lee SS, et al. Eosinophilic meningitis caused by Angiostrongylus cantonensis associated with eating raw snails: correlation of brain magnetic resonance imaging scans with clinical findings. The American Journal of Tropical Medicine and Hygiene 2003; 68 : 281-5.

7. Hwang KP, Chen ER, Clinical studies on angiostrongyliasis cantonensis among children in Taiwan. Southeast Asian Journal of Tropical Medicine and Public Health 1991; 22 (Suppl): 194-9.

8. Wang X, Huang H, Dong Q, Lin Y, Wang Z, et al. A clinical study of eosinophilic meningoencephalitis caused by angiostrongyliasis. Chinese Medical Journal 2002; 115: 1312-5. 


\section{Case reports}

9. Bowden DK. Eosinophilic meningitis in the New Hebrides: two outbreaks and two deaths. The American Journal of Tropical Medicine and Hygiene 1981; 30: 1141-3.

10. Slom TJ, Cortese MM, Gerber SI, Jones RC, Holtz TH, et al. An outbreak of eosinophilic meningitis caused by Angiostrongylus cantonensis in travelers returning from the Caribbean. New England Journal of Medicine 2002; 346: $668-75$.
11. Mentz MB, Graeff-Teixeira C. Drug trials for treatment of human angiostrongyliasis. Revista do Instituto de Medicina Tropical de Sao Paulo 2003; 45: 179-84.

12. Chotmongkol V, Sawanyawisuth K, Thavornpitak Y. Corticosteroid treatment of eosinophilic meningitis. Clinical Infectious Diseases 2000; 31: 660-2. 